\title{
Structural model updating based on metamodel using modal frequencies
}

Jutao Wang, Zhenzhong Liu, Lijun Xue

Tianjin Key Laboratory for Advanced Mechatronic System Design and Intelligent Control, School of Mechanical Engineering, Tianjin University of Technology, Tianjin 300384, China

National Demonstration Center for Experimental Mechanical and Electrical Engineering Education (Tianjin University of Technology)

tjwjt@163.com,liusdcn@gmail.com,meilideshijieali@163.com

ABSTRACT. Modal frequencies are often used in structural model updating, based on the finite element model, while the metamodel approach is often used in the respective optimization process. In this work, the Kriging model is adopted as the metamodel. First, the influence of different correlation functions of Kriging model is inspected, followed by the investigation of the approximate capability of Kriging model by inspecting the approximate accuracy of nonlinear functions. Next, a model updating procedure is proposed, based on the Kriging model, constructed using samples generated via the method of Optimal Latin Hypercube. Finally, a typical frame structure is taken as a case study, demonstrating the feasibility and efficiency of the proposed approach. The results show that, the Kriging model can match the target functions very well, while the finite element model can achieve accurate frequencies and can reliably predict the frequencies after model updating.

KEYwORDS. Model updating; Metamodel; Modal frequency; Optimization.

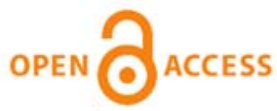

Citation: Wang, J., Liu, Z., Xue, L., Structural model updating based on metamodel using modal frequencies, Frattura ed Integrità Strutturale, 58 (2021) 114-127.

Received: 08.12 .2020

Accepted: 30.05 .2021

Published: 01.07.2021

Copyright: (C) 2021 This is an open access article under the terms of the CC-BY 4.0, which permits unrestricted use, distribution, and reproduction in any medium, provided the original author and source are credited.

\section{INTRODUCTION}

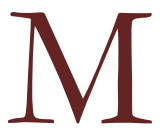

etamodel has been introduced, for some years now, to model updating procedure, due to its fast iteration advantage. Specifically in the structural dynamic model updating case, the metamodel can achieve computational efficiency, when used in place of the Finite Element Model (FEM), during the iteration process of optimization. Ling [1] combined an adaptive Kriging metamodel with importance sampling strategies, to analyze the safety level of timevariant structures. Based on metamodel, Lin [2] proposed an intelligent sampling approach and employed this for the design of a bus body frame. Hamdani [3] used the Kriging model to efficiently solve the expensive mechatronic optimization problem in soldered joints. Metamodel has not only been applied in the updating process, but also in structural optimization [4], uncertainty quantification [5] and damage detection [6], among others. The metamodel can be considered as the description of the input-output relation in the simulation of FEM, where the relation appears obviously nonlinear in dynamic 
simulation cases. The metamodel, offering a strong approximation capability to nonlinear function, is important in dynamic model updating, where fast iteration with accurate metamodeling is needed. In other words, the metamodels are supposed to describe more exact input-output relations in the simulations of FEM.

An adaptive Kriging method was successfully applied to solve the Multidisciplinary Design Optimization (MDO) problem of the geostationary (GEO) satellite [7]. In another study, a multi-objective multidisciplinary design optimization problem of unmanned aerial vehicle wing was solved based on Kriging model [8]. As one of the most common dynamic model updating approaches, modal parameter based model updating is applied on different finite element models for various structures. Bautista-De Castro [9] combined a polynomial chaos expansion metamodel and Sobol's indices for the sensitivity analysis, in the masonry arch bridge case. Based on the modal parameter, the proposed method made Particle Swarm Optimization (PSO) possible to apply, for the model updating of a large-scale railway bridge [10]. In the analysis of vibration characteristics of track structures, Gao [11] carried out 3 model updating schemes, based on the measured modal parameters. In the case of dynamic model updating, the dynamic analysis and objective function of the optimization problem have nonlinear characteristics, which the commonly used Response Surface Method (RSM) [12] is incapable of approximating, therefore the Kriging model is introduced to the dynamic model updating, as a more accurate metamodel. In this article, multidimensional functions are considered as objective functions, for inspecting the approximation capability of the Kriging model. The required training samples, for constructing Kriging model, are obtained by Design of Experiment (DOE). The accuracy of the constructed Kriging model, in respect to the objective functions, is estimated by Root Mean Square Error (RMSE) and determination coefficient. Accordingly, a procedure of model updating is proposed, while a model updating of modal frequencies in a typical frame structure is used as case study.

\section{CHARACTERISTIC OF KRIGING MODEL}

$\mathrm{U}$ nlike the conventional RSM, the Kriging model goes through all the training samples, providing a more accurate approximation than the conventional RSM [13, 14]. The Kriging model is constructed based on the interpolation technique, which can provide evaluation of unknown points. These evaluated values are the unbiased estimation of the least estimated variance of the known points. The Kriging model generally consists of two parts: linear regression and non-parametric sections. The model exhibits statistical characteristics, since the non-parametric section follows random distribution. Thereby the Kriging model can be formulated by polynomial section and random distribution, as follows:

$$
y(x)=F(\beta, x)+q(x)=f^{T}(x) \beta+q(x)
$$

where, $\beta$ is the regression coefficient, $\mathrm{f}(\mathrm{x})$ denotes the polynomial of variable $\mathrm{x}$; and $\mathrm{z}(\mathrm{x})$ denotes the errors of random distribution.

\section{The construction process of the Kriging model}

The Kriging model derives from statistics, based on the theory that the closer the regions, the more similar they are. The function value at an unknown point can be evaluated via weighted summation of the information of known points, located nearby the unknown point. The weighted summation is obtained via minimized variance of the error among the known points. Accordingly, the Kriging model needs to satisfy two requirements: unbiased property and minimizing error estimation. The construction of the Kriging model can then be achieved, as follows:

\section{(i) Unbiased property}

Based on Eqn. (1), the unbiased property is the unbiased estimation which obtains $y\left(x_{0}\right)$ from $\hat{y}\left(x_{0}\right)$, that is, $y\left(x_{0}\right)$ and $\hat{y}\left(x_{0}\right)$ have the same expectation:

$$
\mathrm{E}\left[\hat{y}\left(x_{0}\right)\right]=\mathrm{E}\left[y\left(x_{0}\right)\right]
$$

where, $\hat{y}\left(x_{0}\right)=\sum_{i=1}^{n} \mathrm{w}_{i} y\left(x_{i}\right)$

Eqn. (2) can also be written as follows: 


$$
E\left[\sum_{i=1}^{n} w_{i} y\left(x_{i}\right)\right]=\sum_{i=1}^{n} w_{i} E\left[y\left(x_{i}\right)\right]=\mathrm{E}\left[y\left(x_{0}\right)\right]
$$

where, $w_{\mathrm{i}}$ are the weight coefficients and therefore the unbiased condition followed is as follows:

$$
\sum_{i=1}^{n} w_{i}=1
$$

(ii) Minimizing error estimation (optimal results)

The estimated variance of the unknown point $x_{0}$ can be obtained as

$$
\begin{aligned}
\sigma_{E}^{2} & =E\left[\left(\hat{y}\left(x_{0}\right)-y\left(x_{0}\right)\right)^{2}\right]=E\left[\left(\sum_{i=1}^{n} w_{i} y\left(x_{i}\right)-y\left(x_{0}\right)\right)^{2}\right]= \\
& =\sum_{i=1}^{n} \sum_{j=1}^{n} w_{i} w_{j} C\left(y\left(x_{i}\right), y\left(x_{j}\right)\right)-2 \sum_{i=1}^{n} w_{i} C\left(y\left(x_{i}\right), y\left(x_{0}\right)\right)+C\left(y\left(x_{0}\right), y\left(x_{0}\right)\right)
\end{aligned}
$$

where, $\mathrm{C}$ denotes the matrix of the covariance, while the correlation vector $\mathbf{R}\left(\mathrm{x}_{\mathrm{i}}, \mathrm{x}_{\mathrm{j}}\right)$, based on the unbiased results, can convert Eqn. (5) into the following form:

$$
\sigma_{E}^{2}=2 \sum_{i=1}^{n} w_{i} R\left(x_{i}, x_{0}\right)-\sum_{i=1}^{n} \sum_{j=1}^{n} w_{i} w_{j} R\left(x_{i}, x_{j}\right)-R\left(x_{0}, x_{0}\right), R\left(x_{i}, x_{j}\right)=\sigma^{2}-C\left(x_{i}, x_{j}\right)
$$

The minimization of estimated variance of Eqn. (6), at the unbiased condition, is an extreme-value problem with constraints, while the solution can be obtained based on the Lagrange Multiplier Method, which means:

$$
L\left(w_{i}, \Phi\right)=\sigma_{E}^{2}-2 \Phi\left(\sum_{i=1}^{n} w_{i}-1\right)
$$

where, $\boldsymbol{\Phi}$ is the vector of Lagrange Multiplier, the partial derivatives of $w_{\text {i }}$ and $\boldsymbol{\Phi}$ to L are set to 0, deriving:

$$
\left\{\begin{array}{l}
\frac{\partial L}{\partial w_{i}}=2 \sum_{j=1}^{n} w_{j} R\left(x_{i}, x_{j}\right)-2 R\left(x_{i}, x_{0}\right)-2 \Phi=0 \\
\frac{\partial L}{\partial \Phi}=-2\left(\sum_{i=1}^{n} w_{i}-1\right)=0
\end{array}\right.
$$

after adjustment, the form is:

$$
\left\{\begin{array}{l}
\sum_{j=1}^{n} w_{j} R\left(x_{i}, x_{j}\right)-\Phi=R\left(x_{i}, x_{0}\right) \\
\sum_{i=1}^{n} w_{i}=1
\end{array}\right.
$$

The weight coefficient $w_{\mathrm{i}}$ and Lagrange coefficient $\boldsymbol{\Phi}$ can be obtained via solving linear Eqn. (9), while the evaluated value of $\hat{y}\left(x_{0}\right)$ at unknown point $x_{0}$ can be derived, based on Eqn. (2). Eqn. (9) can be written as a matrix: 


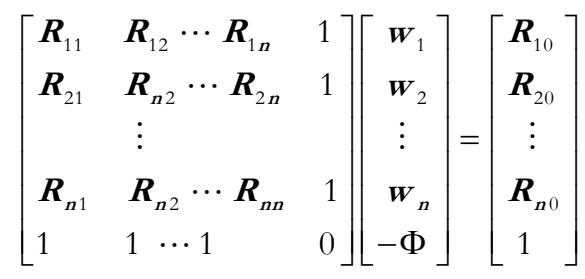

where, $\mathbf{R}_{\mathrm{ij}}=\mathbf{R}\left(x_{\mathrm{i}}, x_{\mathrm{i}}\right)$.

The values of $w$ i and $\boldsymbol{\Phi}$ can be solved directly by reversing the matrix Eqn. (10), where the matrix $\mathrm{r}_{\mathrm{ij}}$ of correlation coefficient can be obtained independently, in advance. Accordingly, the construction steps of Kriging model can be summarized as follows:

Step 1: the distance and $\mathrm{R}\left(x_{\mathrm{i}}, x_{\mathrm{j}}\right)$ between every two known points are calculated successively.

Step 2: the correlation function is selected based on the value of $\mathrm{d}_{\mathrm{ij}}$ and $\mathbf{R}_{\mathrm{ij}}$, as provided from Step 1.

Step 3: $\mathrm{R}_{\mathbf{i} 0}$ of the unknown point $\chi_{0}$ is calculated based on the selected correlation function.

Step 4: the optimal value of the weight coefficient $w_{i}$ is obtained based on Eqn. (10).

Step 5: the weighted array of the known points, based on the $w_{i}$, is used to evaluate the unknown point $x_{0}$.

The matrix of the correlation coefficient is fixed once the left side of Eqn. (10) is evaluated. Therefore, the column vector of the right side equation is the only calculation held separately, thus the values of the unknown points can be obtained.

The parameter $\theta$ is introduced into the function $\mathrm{R}\left(x_{1}, x_{\mathrm{i}}\right)$ for more flexibility. The function $\mathrm{y}(x)$ follows normal distribution, since $\mathrm{z}(x)$ follows normal distribution in Eqn. (1), while the $\theta$ value is obtained by maximizing the likelihood estimation of $\mathrm{y}(x)$, which can assure that $\mathrm{y}(x)$ goes through the known points with maximum probability. The equation form, after logarithm to likelihood function of $\mathrm{y}(x)$, for reasons of facilitating calculation, can be given as:

$$
\ln [\operatorname{Lh}(R \mid y)]=-\frac{n_{s}}{2} \ln \left(\sigma^{2}\right)-\frac{1}{2} \ln (|R|)-\frac{\left(Y-F^{T} \beta\right)^{T} R^{-1}\left(Y-F^{T} \beta\right)}{2 \sigma^{2}}
$$

After partial derivative and maximum likelihood estimation, the construction of Kriging model focuses on the maximum solution of $\theta$ value [16].

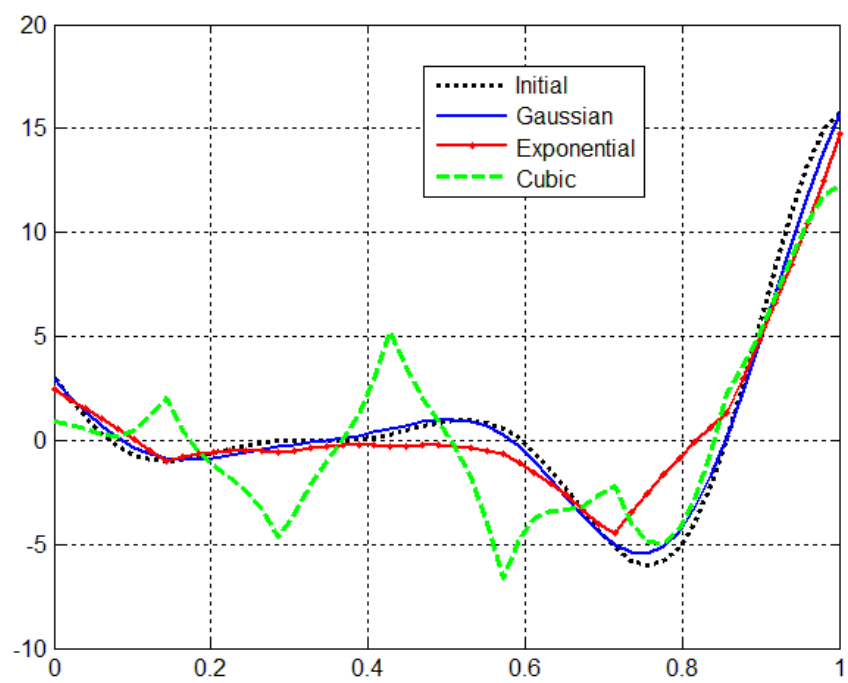

Figure 1: The approximation result by Kriging model using different correlation functions.

\section{The types of correlation function}

The correlation function $\mathrm{R}_{\mathrm{ij}}$ describes the correlation between samples, being stronger among the closer together samples. The evaluation of the unknown point depends on the stronger correlations. The main types of correlation functions are: Linear, Spherical, Gaussian, Exponential and Cubic. Each of the functions shows its own advantages, in specific applications, e.g. Exponential function is more suitable for biology, while the application of Gaussian function is more 
extensive $[17,18]$. A comparative graph is illustrated in Fig. 1, to show the approximation ability to a one-dimension function (Eqn. (12)). The "initial" line represents the one-dimension function (objective function), the other 3 lines represent the approximation of different correlation functions. Fig. 1 shows that, the smoothest curve is constructed based on Gaussian function, which is suitable for optimization algorithms.

$$
f(x)=(6 x-2)^{2} \sin (12 x-4)
$$

\section{SELECTION OF TRAINING SAMPLES}

7 he training samples (known points) are usually obtained via the DOE method and used to construct the Kriging model. The matrix of design parameters is generated by the DOE method (a planning method), in which the response of the design matrix can reflect the properties of the designed object, such as the sensitivities and interactions of design parameters. The design matrix consists of the descriptions of the samples distribution, in which the uniformity and orthogonality can affect the approximate accuracy of the derived Kriging model. The commonly used sampling methods are: All Factors, Orthogonal Array, Central Composite, Latin Hypercube and Optimal Latin Hypercube $(\mathrm{OLH})$. Each parameter can be involved and given average values by the Latin Hypercube method, making it possible for fewer samples to fill the design domain. Based on Latin Hypercube method, OLH method can minimize the "ununiformity" of distribution of the samples, via Central $\mathrm{L}_{2}\left(\mathrm{CL}_{2}\right)$ criterion $[19,20]$. The advantage of the $\mathrm{CL}_{2}$ criterion is illustrated in Fig. 2 and its expression is given by Eqn. (13). Each dot in Fig. 2 represents a sample, while the grid represents the design domain. Obviously, in the same small number of samples, the distribution of samples in OLH is more uniform. Briefly stating that, in constructing Kriging model, the OLH method is more suitable in cases of fewer samples.

$$
\begin{aligned}
C L_{2}(X) & =\left(\frac{13}{12}\right)^{2}-\frac{2}{n} \sum_{i=1}^{n} \prod_{k=1}^{m}\left(1+\frac{1}{2}\left|x_{i k}-0.5\right|-\frac{1}{2}\left|x_{i k}-0.5\right|^{2}\right)+ \\
& +\frac{1}{n^{2}} \sum_{i=1}^{n} \sum_{j=1}^{n} \prod_{k=1}^{m}\left(1+\frac{1}{2}\left|x_{i k}-0.5\right|-\frac{1}{2}\left|x_{j k}-0.5\right|-\frac{1}{2}\left|x_{i k}-x_{j k}\right|\right)
\end{aligned}
$$

where, $\mathrm{n}$ denotes the times of sampling, $\mathrm{m}$ denotes the number of design variables, $\mathrm{x}_{\mathrm{ik}}$ denotes the $\mathrm{i}^{\text {th }}$ sampling of design variable $\mathrm{x}_{\mathrm{k}}$.
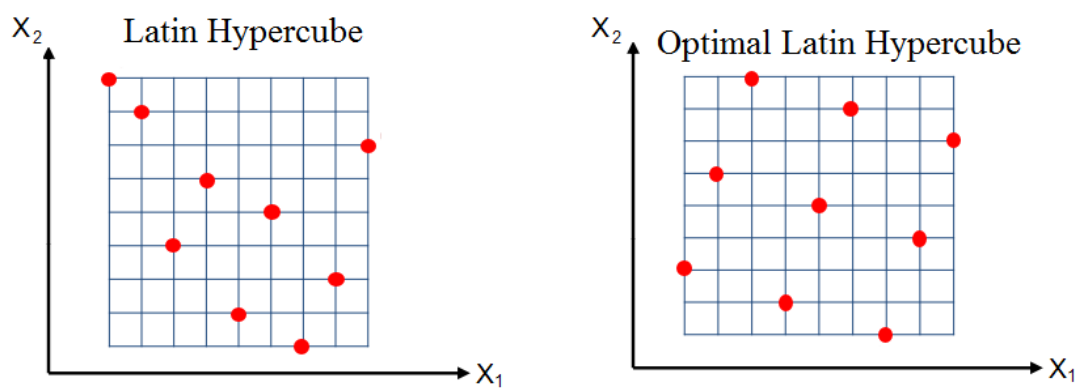

Figure 2: The comparison of Latin Hypercube to OLH.

\section{TEST FOR KRIGING MODEL}

$\mathrm{M}$ etamodel is seen as an approximation to the target model (objective function) and the approximation accuracy must be determined, before replacing the target model. In this section, the approximation capability of Kriging model is investigated, by interpolating standard test functions. The Kriging model is constructed based on the training samples, derived from the test functions. Next, the check points are re-sampled, providing different sets from the training samples. The check points are used to compare the corresponding output of the Kriging model to the target model. The discrepancies (i.e. error) revealed by the comparison are estimated by RMSE method or using the determination coefficient $\left(\mathrm{R}^{2}\right)$, to describe the approximation capability. 
The deviations between target values and approximated values are estimated by RMSE method, deriving an average value of the observed discrepancies, which the lower it is, the smaller the error. In engineering, the error is usually supposed to be less than 0.1 (RMSE $<0.1)$. The value of $\mathrm{R}^{2}$ is used to estimate the similarity between the target model and the metamodel, whereas the greater the value, the more similar the 2 models are. The range of $\mathrm{R}^{2}$ is between 0 and 1 , while the value is supposed to be greater than $0.9\left(\mathrm{R}^{2}>0.9\right)$. The expressions of RMSE and $\mathrm{R}^{2}$ method are as follows:

$$
R M S E=\frac{1}{k \overline{k y}} \sqrt{\sum_{i=1}^{k}\left(y_{i}-\hat{y}_{i}\right)^{2}}, \quad \mathrm{R}^{2}=1-\frac{\sum_{i=1}^{k}\left(y_{i}-\hat{y}_{i}\right)^{2}}{\sum_{i=1}^{k}\left(y_{i}-\bar{y}\right)^{2}}
$$

Where, $\mathrm{k}$ denotes the number of test points, $\overline{\mathrm{y}}$ denotes the average value of target model response, $\mathrm{yi}_{\mathrm{i}}$ denotes the response of the target model at the test points, $\hat{y}_{i}$ denotes the response value of the Kriging model at the test points.

One of the commonly used functions, Branin Function is applied to inspect the approximation capability of Kriging model. Fig. 3 shows that, Branin Function is an asymmetric function of nonlinear function, with input variables ranges of [-5,10] and $[0,15]$. The expression is as follows:

$$
y(x)=\left(x_{2}-\frac{5.1 x_{1}^{2}}{4 \pi^{2}}+\frac{5 x_{1}}{\pi}-6\right)^{2}+10\left(1-\frac{1}{8 \pi}\right) \cos \left(x_{1}\right)+10, \quad\left(-5 \leq x_{1} \leq 10,0 \leq x_{2} \leq 15\right)
$$

The number of samples population are 10, 15 and 20 respectively, sampled by the OLH method respectively. Based on the samples, the contrasts between the constructed Kriging model and Branin Function are illustrated in Figs. 4(a), 4(b) and $4(\mathrm{c})$, respectively. The largest error appears in the range of $(-5,0)$, while it is apparently decreasing as the number of samples increases. There is a large edge error illustrated in Fig. 4(a), but the Kriging model also has a very similar shape to the one of the objective function. Better consistencies appear in Figs. 4(b) and 4(c), whereas the main errors at the boundaries are far from the samples.

The values of RMSE and $\mathrm{R}^{2}$, at the check points, are used to test the approximation accuracy of the Kriging model. 5 samples are sampled, using Latin Hypercube method, as check points, while 3 errors at the check samples of each Kriging model are compared to the target function (Fig. 5). It is evident that, the error is decreasing when the number of training samples is growing. The values of RMSE and $\mathrm{R}^{2}$, in the 3 different training samples, are listed in Table 1 , at the check samples. The results show that, the Kriging models have a better approximation accuracy, when constructed by 15 samples and 20 samples. However, there is little improvement in accuracy, when using the 20 samples, which means that 15 training samples is an adequate number in this test function.

\begin{tabular}{cccc}
\hline Sample number & 10 & 15 & 20 \\
RMSE value & 0.1366 & 0.0467 & 0.0433 \\
$\mathrm{R}^{2}$ value & 0.9350 & 0.9924 & 0.9935 \\
\hline
\end{tabular}

Table 1. The errors in different sample sets of Kriging model in different estimations

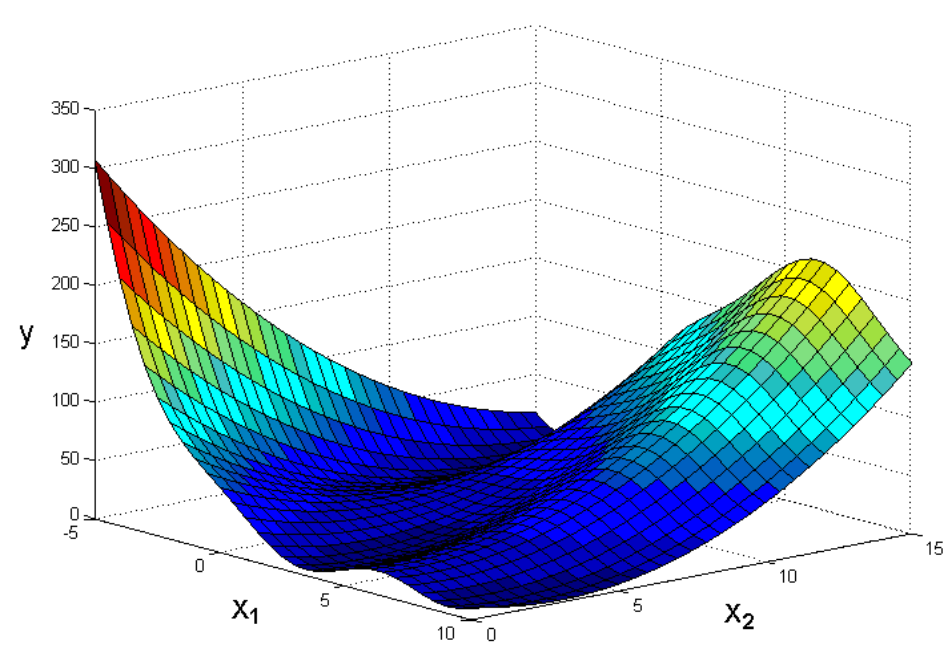

Figure 3: The 3 dimensional plot of Branin function. 


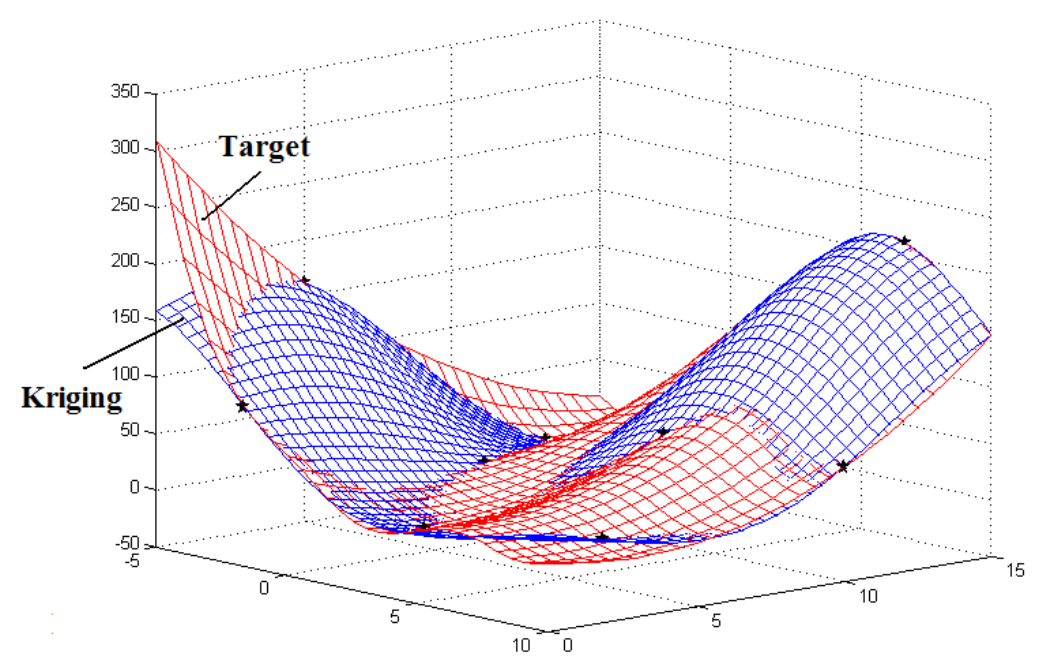

(a)

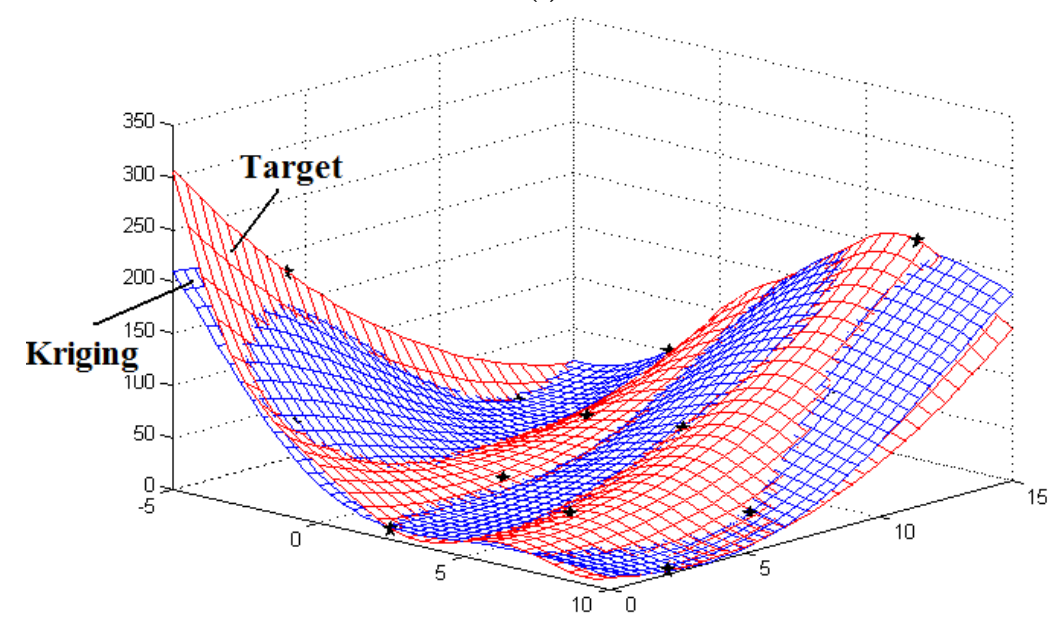

(b)

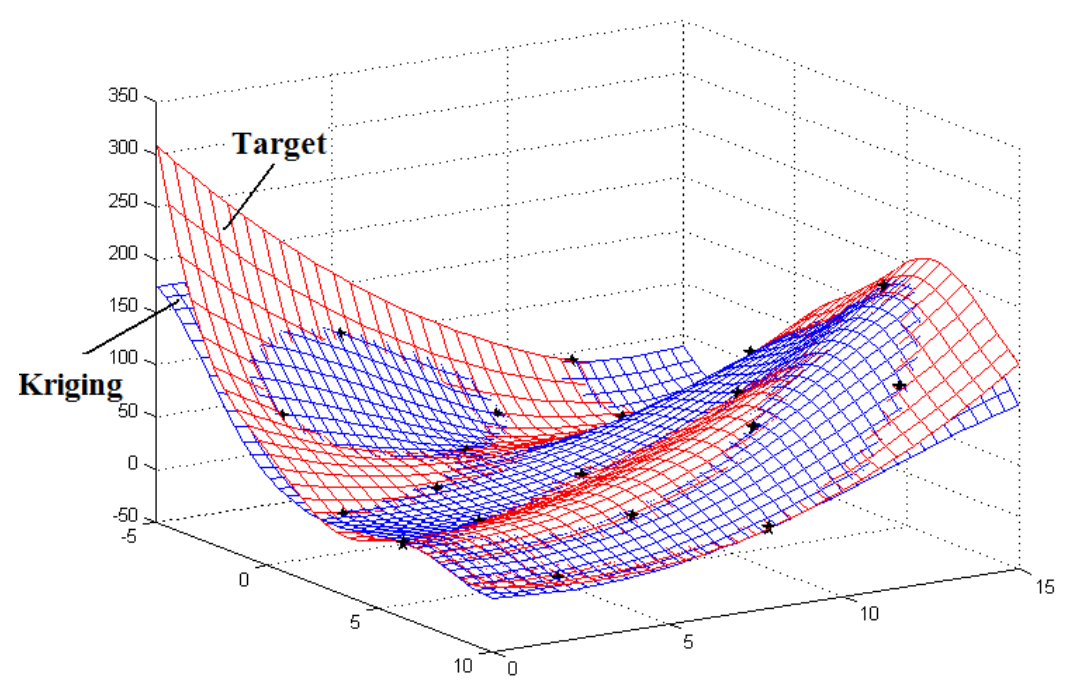

(c)

Figure 4: The Kriging model for Branin Function for different number of samples: (a) 10 samples, (b)15 samples, (c) 20 samples. 


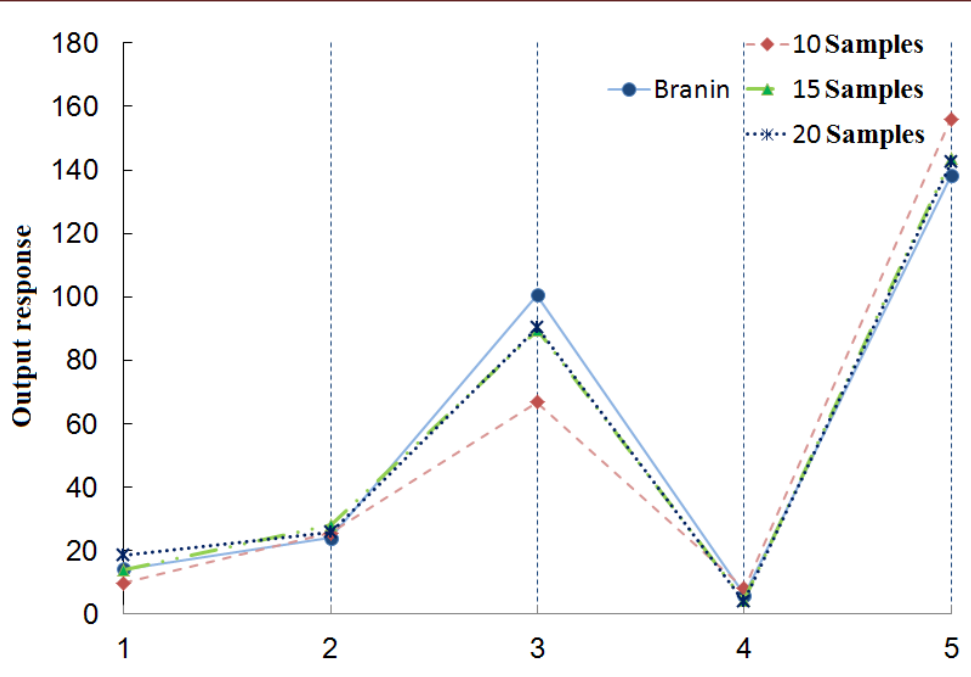

Figure 5: The error at the check points.

\section{THE DYNAMIC MODEL UPDATING BASED ON KRIGING MODEL}

\section{The construction of objective function}

$\mathrm{M}$ odel updating aims to make the updated FEM have the same behavior as the real experiment, as much as possible. Model updating is a mechanical inverse problem, where the FEM is calibrated, based on the known response of the experiment model. The objective function is formulated based on the response of FEM and the experiment, while the model updating can then be converted to constrained optimization problem. The form of a commonly used objective function is formulated as follows:

$$
\left\{\begin{array}{l}
\min F(x)=\sum_{i=1}^{b} w_{i}\left(\frac{\mathrm{R}_{t}^{i}-\mathrm{R}_{a}^{i}}{\mathrm{R}_{t}^{i}}\right)^{2} \\
\text { s.t. } x_{j}^{d} \leq x_{j} \leq x_{j}^{u} \quad(j=1,2, \ldots, n)
\end{array}\right.
$$

Where, $w_{i}$ denotes the weight coefficient, $R_{a}$ denotes the response of the FEM, $R_{t}$ denotes the response of the experiment model, $x_{j}$ denotes design parameters, $x_{d}$ denotes the lower bound of design parameter and $x_{u}$ denotes the upper bound.

\section{The process of dynamic model updating based on Kriging model}

The structural features and response types are taken into account for the construction of a suitable FEM, requiring rational simplification and parameterization and so on. The DOE of the FEM is carried out to generate the training samples for constructing the respective Kriging model. The objective function is established based on the response deviation between the experiment and the FEM, while then the optimal value of design parameters is obtained by solving the optimization problem. The optimized parameters are used to modify the FEM and produce the updated FEM. The process of dynamic model updating, based on Kriging model, is plotted in Fig. 6 and summarized below:

Step 1: Constructing original FEM based on the experiment.

Step 2: Parameterizing the FEM and selecting the design parameters to be optimized.

Step 3: DOE is carried out, based on the FEM, while the design matrix is obtained using the OLH method for design parameters. The training samples are generated by calculating the response on each level of the DOE.

Step 4: The relative parameters are determined based on the training sample, to construct the Kriging model.

Step 5: The approximation accuracy of the constructed Kriging model is estimated and when the accuracy satisfies the requirements the process goes to the next step, otherwise there is another iteration starting from Step 3.

Step 6: The response between FEM and experiment are used to establish the objective function, whose design variables are the parameters to be modified.

Step 7: The Kriging model is iterated using a global optimization algorithm, to get the optimal value of design parameters, whereas the iteration will continue until the end condition is satisfied. 
Step 8: The optimal parameters are used to calibrate the FEM, while the model updating is completed after checking the update effect.

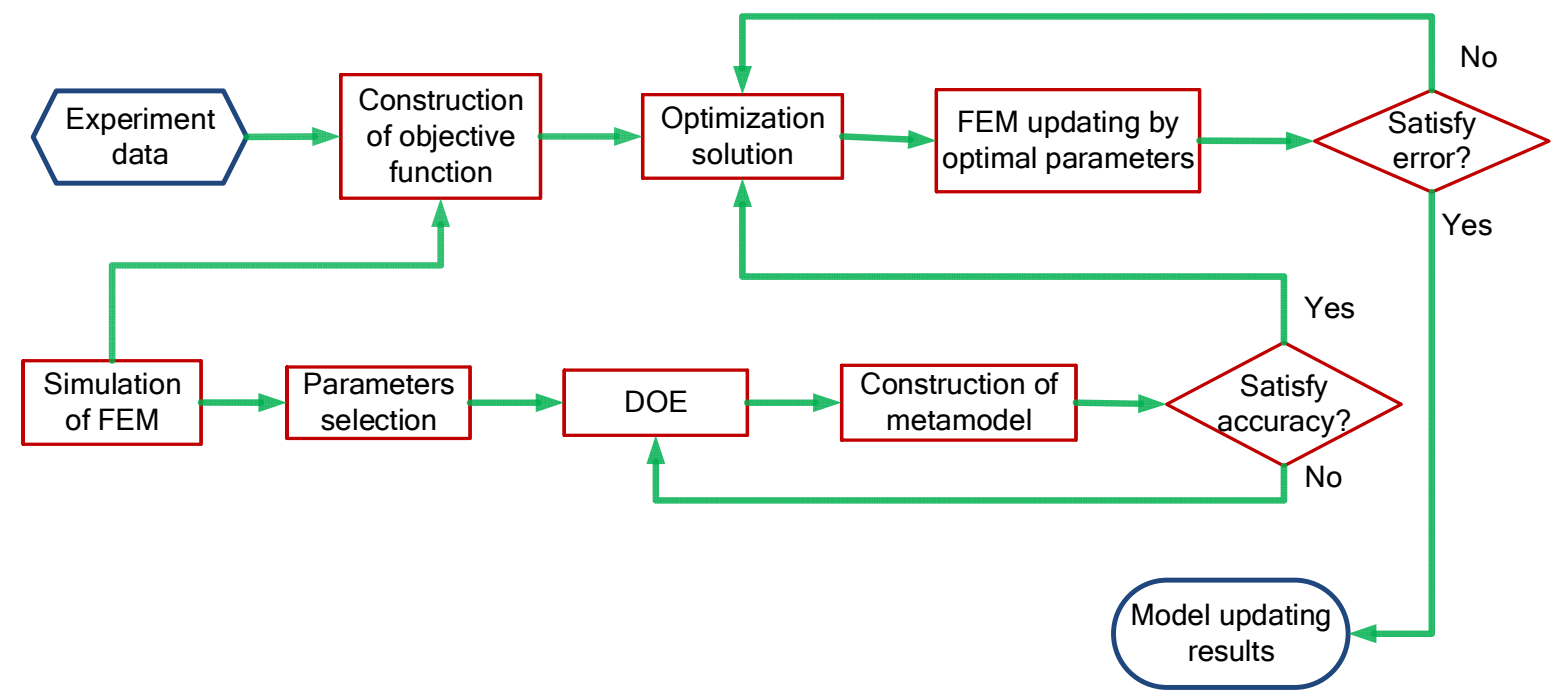

Figure 6: Flow chart of the process of model updating.

\section{CASE STUDY}

$\mathrm{F}$ rame structure is one of the main bearing structures in engineering, such as in the vehicle chassis, or in some civil structure. The dynamic response of the frame structure is very important to the dynamic behavior of the overall structure. The modal control is one of the necessary considerations of dynamic design. The simulation model usually needs model updating, based on the modal frequencies. In this section, an experiment with a typical frame structure [21] is used, to demonstrate the efficiency of the proposed model updating process.

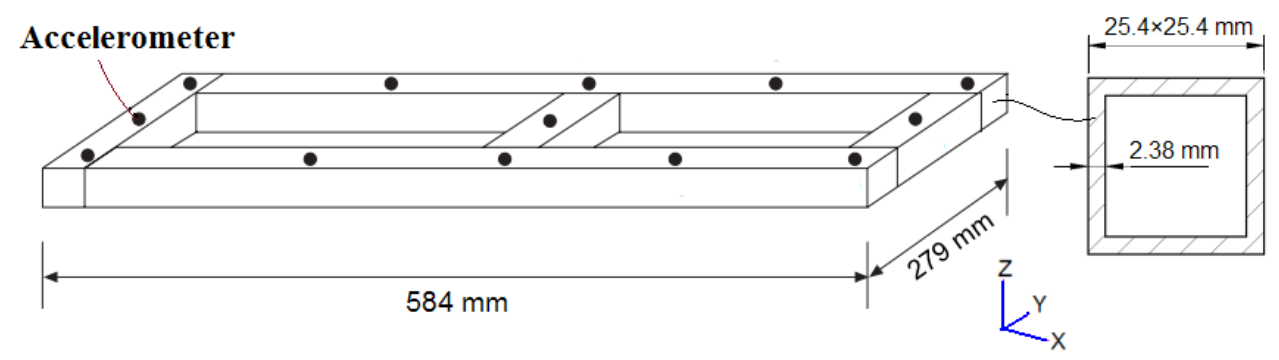

(a)

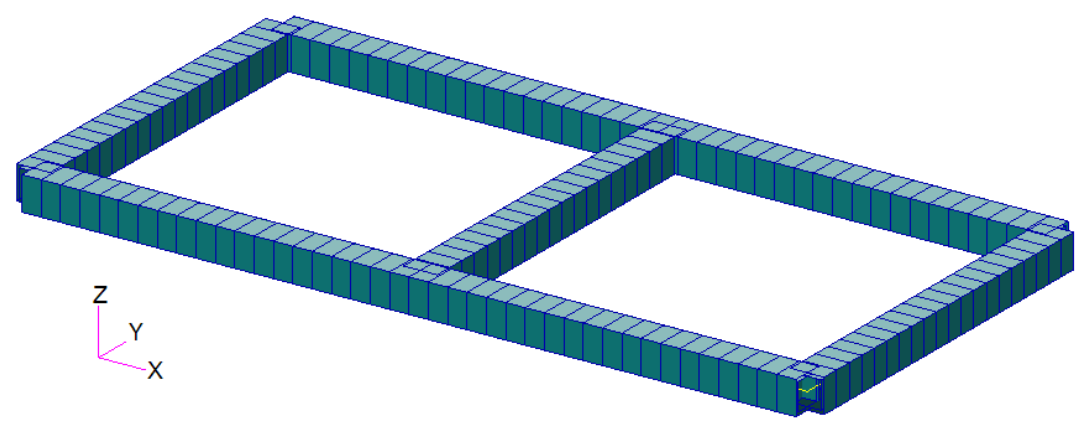

(b)

Figure 7: The frame structure: (a) The frame geometry for experiment, (b) The FEM. 
The construction of FEM and Kriging model

The frame structure (Fig. 7(a)) is assembled by aluminum alloy profile, of $25.4 \mathrm{~mm}$ square side length and $2.38 \mathrm{~mm}$ wall thickness. The modal experiment is performed by hammer testing, where the frame is suspended freely. The first 5 frequencies of the obtained results are listed in the second column of Table 2 . The frame is assembled by 5 beams, while its simplified FEM is constructed by beam elements, similarly to the initial FEM of 140 elements and 139 nodes (Fig. 7(b)). The software Patran has been employed. All elements have same material properties as the initial given values: $\mathrm{E}=71000 \mathrm{MPa}$ (elastic modulus), $\mu=0.3$ (Poisson ratio), $\varrho=2700 \mathrm{Kg} / \mathrm{m}^{3}$ (density).

After free modal analysis of the initial FEM, the first 5 frequencies of the modal results are listed in the third column of Table 2. The initial errors between the FEM and the experimental model are listed in the 4th column of Table 2. Obviously, all errors are too large to be accepted, except the case of the second frequency, whereas all the simulation values are higher than the experimental values. This means that, the initial FEM has internal defects and needs to be calibrated through the process of model updating. Regarding the parameters to be updated, the geometry dimensions are easily measured and should not be selected as parameters to be updated, whereas the material properties are usually coming from handbooks and may easily include errors, so these three properties are selected as parameters to be updated by the model updating process.

\begin{tabular}{cccc}
\hline Order & Experiment $(\mathrm{Hz})$ & $\operatorname{FEM}(\mathrm{Hz})$ & Initial error $(\%)$ \\
1 & 226.8 & 246.13 & 8.52 \\
2 & 275.2 & 283.61 & 3.06 \\
3 & 537.4 & 574.38 & 6.88 \\
4 & 861.5 & 911.46 & 5.80 \\
5 & 974.8 & 1062.70 & 9.02 \\
\hline
\end{tabular}

Table 2. Initial value of modal frequencies of experiment and FEM

In order to demonstrate the efficiency of the model updating process, based on Kriging model, the five frequencies of the modal experiment are divided into two groups, during model updating. The first three frequencies are selected as the updating objective and are named "updating group". On the other hand, the last two frequencies, which will not be updated during model updating, are named "predictive group". The deviations between the experiment and simulation of the first three frequencies are considered as the output response of the DOE, while the expression is as follows:

$$
R(3)=\left|F_{t}^{1}-f_{a}^{1}\right|+\left|F_{t}^{2}-f_{a}^{2}\right|+\left|F_{t}^{3}-f_{a}^{3}\right|
$$

where, $F_{t}^{i}$ denotes the experiment frequencies, $f_{a}^{i}$ denotes the simulated frequencies of FEM.

The three material properties are selected as the updating parameters and are considered as the factors of the DOE. The artificial range of each factor is from $90 \%$ to $110 \%$ of their initial values, while 20 training samples are generated by OLH. Next, the $\theta$ values are obtained as: $\theta_{\mathrm{E}}=0.17174, \theta_{\mu}=0.02724$ and $\theta_{\varrho}=0.17027$. The Kriging model in the $(\mathrm{E}, \varrho)$ dimension is plotted in Fig. 8, with the $\mu$ parameter fixed at its initial value. Five check samples are re-sampled by Latin Hypercube method, to estimate the accuracy. The comparison of response values between the FEM and Kriging model, at each of the check samples, are plotted in Fig. 9. Evaluation produces the values of RSME $=0.375$ and $\mathrm{R}^{2}=0.993$, while it can be considered that the Kriging model shows good accuracy for replacing the FEM in the next process of optimization.

\section{Model updating results and discussion}

Minimizing the error between the FEM value and experimental value of the first three frequencies is the optimization objective. The sum of the error at each frequency is considered as the objective function, whose weight coefficient is set to 1 , since the frequencies have the same order of magnitude. The form of the objective function remains the same as in Eqn. (17), while the input variables (updating parameters) have the same value ranges as in the DOE. Based on the constructed Kriging model, the optimal values of each updating parameter are obtained by Multi-Island Genetic Algorithm as: $\mathrm{E}=67008.5 \mathrm{MPa}$ (elastic modulus), $\mu=0.3122$ (Poisson ratio), $\varrho=2885.6 \mathrm{Kg} / \mathrm{m}^{3}$ (density). The FEM is then calibrated according to the optimal parameters values, while the updated first 5 frequencies are listed in the 3rd column of Table 3. The errors of the updating group and the predictive group evidently decrease, excluding the 2 nd frequency case. 


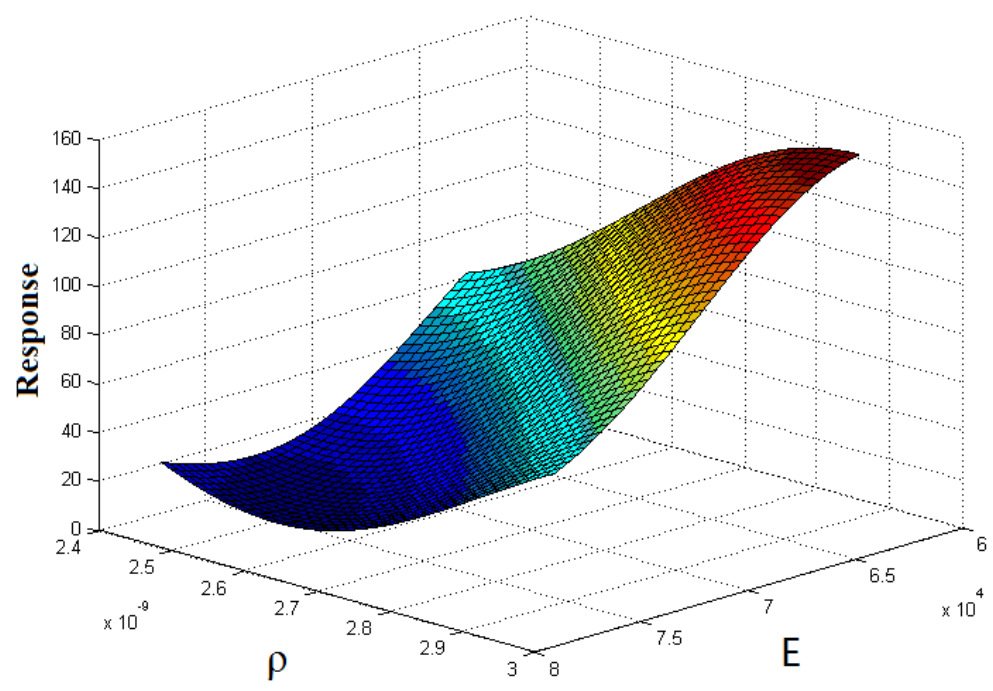

Figure 8: The Kriging model in $(\mathrm{E}, \mathrm{Q})$ dimension.

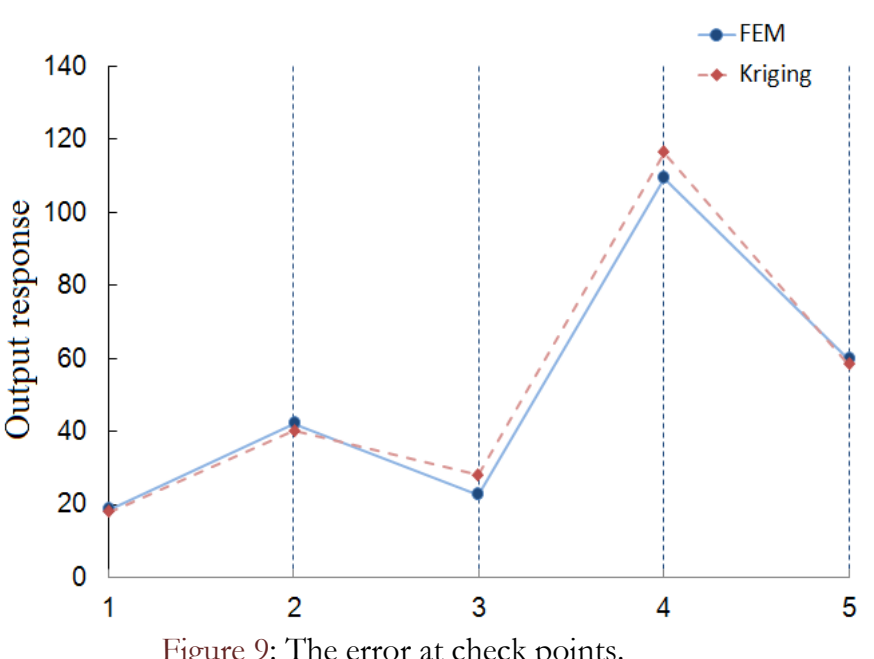

As illustrated in Fig. 8, the minimum is close to the boundary of the design domain, while the global optimal results are possibly located outside the design domain. Therefore, the design domain (value ranges of design parameters) is further expanded, to find out if there is a better optimal result. A new DOE is then operated in a wider design domain, which means each factor ranges between $80 \%$ to $120 \%$ of its initial values, while then 20 training samples are generated by OLH. Next, the corresponding $\theta$ values are obtained as: $\theta_{\mathrm{E}}=0.32992, \theta_{\mu}=0.00010$ and $\theta_{\varrho}=0.33473$. Consequently, a new Kriging model in the $(\mathrm{E}, \varrho)$ dimension is constructed and plotted (Fig. 10), where the minimum location is promoted so that it is not very close to the boundary. The estimation of the accuracy of the new constructed Kriging model is illustrated in Fig. 11, while the corresponding values are the following: RMSE $=0.0584$ and $\mathrm{R}^{2}=0.9894$. The accuracy declines slightly after expanding the design domain, because the design domain is expanded but the number of samples remains the same.

Based on the Kriging model in Fig. 10, the optimal parameters values, upon completion of the solution process, are: $\mathrm{E}=$ $72384.4 \mathrm{MPa}, \mu=0.3486, \varrho=3068.2 \mathrm{Kg} / \mathrm{m}^{3}$. The corresponding result of model updating is listed in the 5 th column of Table 3 , where it is evident that, the errors of the 1 st, 2nd and 4th frequencies decline and the other errors slightly increase, while the errors still meet the relative engineering requirements. The results listed in the last two lines in Table 3 are the predicted values, where the 4th and 5th frequencies are updated correctly, meaning that the two Kriging models have a good predictive capability.

Moreover, for comparison reasons, the commonly used RSM with second-order polynomial is constructed, based on the 20 training samples, previously generated in the expanded design domain. The updated results are listed in the 7 th column of Table 3, where most of the error is larger than the other three groups of errors, except at the 2nd frequency. Meanwhile, 
the optimization without metamodel is applied to the FEM directly (the parameters are in the expanded design domain), and the updated result is listed in the penultimate column of Table 3, the error is almost at the same level as the proposed method. This can lead to the conclusion that the Kriging model shows advantages in the specific dynamic model updating.

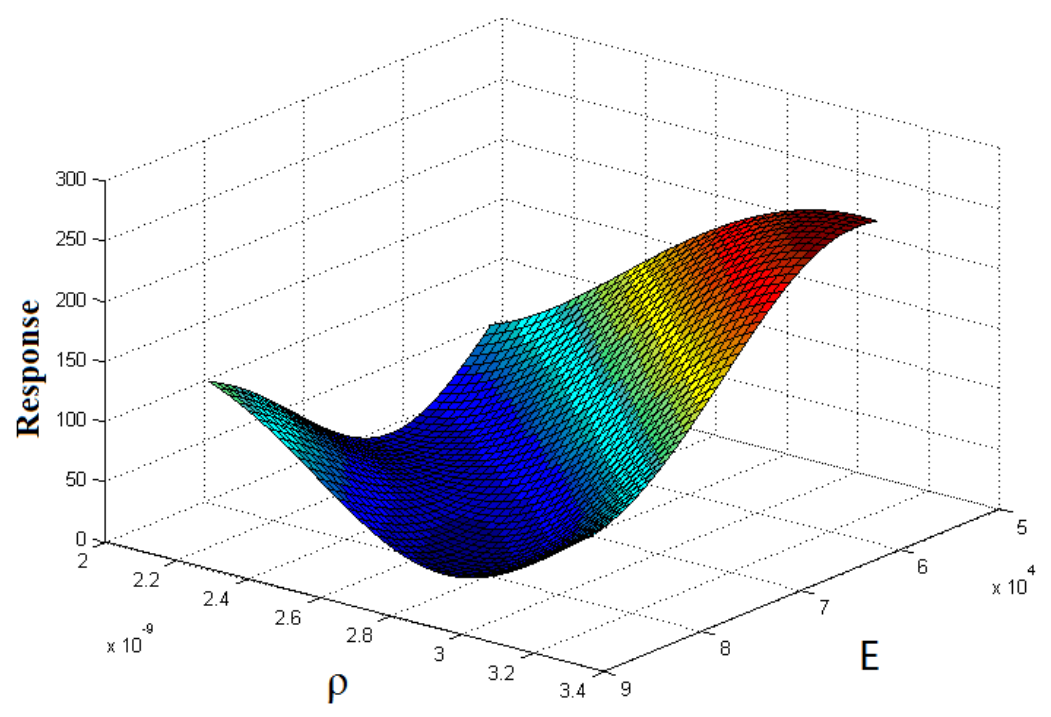

Figure 10: The Kriging model in (E, @) dimension

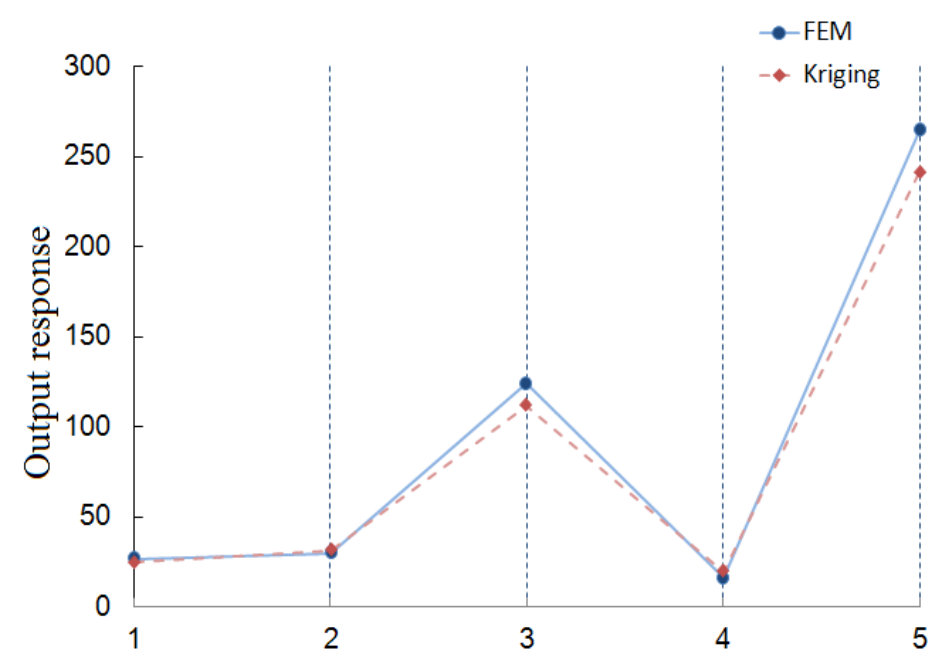

Figure 11: The error at checking points.

\begin{tabular}{|c|c|c|c|c|c|c|c|c|c|}
\hline \multirow[b]{2}{*}{$\begin{array}{c}\text { Orde } \\
r\end{array}$} & \multirow[b]{2}{*}{$\begin{array}{c}\text { Experime } \\
\mathrm{nt} \\
(\mathrm{Hz})\end{array}$} & \multicolumn{2}{|c|}{ $\pm 10 \%$ variation } & \multicolumn{2}{|c|}{ $\pm 20 \%$ variation } & \multicolumn{2}{|l|}{ RSM } & \multicolumn{2}{|c|}{ Directly } \\
\hline & & $\begin{array}{c}\text { After } \\
\text { updated } \\
(\mathrm{Hz})\end{array}$ & $\begin{array}{c}\text { Error } \\
(\%)\end{array}$ & $\begin{array}{c}\text { After } \\
\text { updated }(\mathrm{Hz} \\
)\end{array}$ & $\begin{array}{c}\text { Error } \\
(\%)\end{array}$ & $\begin{array}{c}\text { After } \\
\text { updated }(\mathrm{Hz} \\
)\end{array}$ & $\begin{array}{c}\text { Error } \\
(\%)\end{array}$ & $\begin{array}{c}\text { After } \\
\text { updated }(H \\
\text { z) }\end{array}$ & $\begin{array}{c}\text { Erro } \\
r \\
(\%)\end{array}$ \\
\hline 1 & 226.8 & 230.39 & 1.58 & 229.57 & 1.22 & 232.14 & 2.35 & 228.18 & 0.61 \\
\hline 2 & 275.2 & 266.46 & -3.18 & 268.50 & -2.43 & 272.44 & -1.00 & 267.79 & -2.69 \\
\hline 3 & 537.4 & 538.66 & 0.23 & 539.80 & 0.45 & 546.80 & 1.75 & 537.46 & 0.01 \\
\hline 4 & 861.5 & 856.08 & -0.63 & 861.81 & 0.036 & 874.20 & 1.47 & 859.26 & -0.26 \\
\hline 5 & 974.8 & 998.06 & 2.39 & 1004.48 & 3.04 & 1018.85 & 4.52 & 1001.43 & 2.73 \\
\hline
\end{tabular}

Table 3. Comparison before and after model updating 


\section{CONCLUSION}

$\mathrm{M}$

Odel updating method based on metamodel is studied in this article, where the advantages of different correlation functions and different sampling methods are compared, so that Gaussian function and OLH are selected for the Kriging model. First, the approximation accuracy of Kriging model to the objective function (Branin Function) is inspected in different number of samplings. The errors between the objective function and Kriging models are quantified by RSME and $\mathrm{R}^{2}$ criteria, based on the check points. After inspection, all the constructed Kriging models can achieve RMSE $<5 \%, \mathrm{R}^{2}>0.99$, which means Kriging model exhibits a good capability to approximate the nonlinear function. According to the characteristic of dynamic model updating, Kriging model is considered as the metamodel, to replace the FEM in the iterations, during the optimization process. Based on the proposed approach, a typical frame structure is used as the case study, where the FEM is established by beam elements. The DOE response and optimization objective are constructed based on the errors of modal frequencies between the FEM and the experiment. After model updating, the modal errors of the FEM are reduced, while the updated results are better than the results of RSM, while the updated FEM shows considerable prediction ability. Therefore, the efficiency of dynamic model updating, using the proposed method, is demonstrated and further lays a foundation for subsequent relative research.

\section{ACKNOWLEDGEMENT}

7 his work was supported in part by the National Natural Science Foundation of China under Grant 61873188, and in part by the Tianjin Science and Technology Plan Projects under Grant 18ZXZNGX00360.

\section{REFERENCE}

[1] Ling, C., Lu, Z. (2020). Adaptive Kriging coupled with importance sampling strategies for time-variant hybrid reliability analysis, Applied Mathematical Modelling, 77(2), pp. 1820-1841. DOI: 10.1016/j.apm.2019.08.025.

[2] Lin, C., Gao, F., Bai, Y. (2018). An intelligent sampling approach for metamodel-based multi-objective optimization with guidance of the adaptive weighted-sum method, Structural \& Multidisciplinary Optimization, 58, pp.1047-1060. DOI: $10.1007 / \mathrm{s} 00158-017-1793-2$

[3] Hamdani, H., Radi, B., Hami, A. E. (2019). Metamodel assisted evolution strategies for global optimization of solder joints reliability in embedded mechatronic devices, Microsystem Technologies, 25, pp. 3801-3812. DOI: $10.1007 / \mathrm{s} 00542-019-04520-1$

[4] Shu, L., Jiang, P., Zhou, Q., et al. (2018). An On-line Variable Fidelity Metamodel Assisted Multi-Objective Genetic Algorithm for Engineering Design Optimization, Applied Soft Computing, 66, pp. 438-448.

DOI: 10.106/j.asoc.2018.02.033.

[5] Rodriguez, S., Ludkovski, M. (2020). Probabilistic bisection with spatial metamodels, European Journal of Operational Research, 286(2), pp. 588-603. DOI: 10.1016/j.ejor.2020.03.049.

[6] Ghiasi, R., Ghasemi, M. R., Noori, M. (2018). Comparative studies of metamodeling and AI-Based techniques in damage detection of structures, Advances in Engineering Software, 125, pp. 101-112. DOI: 10.1016/j.advengsoft.2018.02.006.

[7] Shi, R., Liu, L., Long, T., et al. (2019). Filter-based adaptive Kriging method for black-box optimization problems with expensive objective and constraints, Computer Methods in Applied Mechanics \& Engineering, 347, pp. 782-805. DOI: 10.1016/j.cma.2018.12.026.

[8] Zadeh, P. M., Sayadi, M. (2019). An efficient metamodel-based multi-objective multidisciplinary design optimization framework, Applied Soft Computing, 74, pp. 760-782. DOI: 10.1016/j.asoc.2018.09.014.

[9] Bautista-De Castro, A., Javier Sanchez-Aparicio, L. (2019). Carrasco-Garcia P., et al. A multidisciplinary approach to calibrating advanced numerical simulations of masonry arch bridges, Mechanical systems and signal processing, 129, pp. 337-365. DOI: 10.1016/j.ymssp.2019.04.043.

[10] Tran-Ngoc, H., He, L., Reynders, E., et al. (2020). An efficient approach to model updating for a multispan railway bridge using orthogonal diagonalization combined with improved particle swarm optimization, Journal of Sound and Vibration, 476. Pp. 1-18. DOI: 10.1016/j.jsv.2020.115315.

[11] Gao, L., An, B., Xin, T., et al. (2020). Measurement, analysis, and model updating based on the modal parameters of high-speed railway ballastless track, Measurement, 161, pp. 1-14. DOI: 10.1016/j.measurement.2020.107891. 
[12] Gunst, R. F. (2008). Response Surface Methodology: Process and Product Optimization Using Designed Experiments, Technometrics, 38(3), pp. 284-286. DOI: 10.1080/00401706.1996.10484509.

[13] Fang, J., Gao, Y., Xu, C., et al. (2014). Multi-body dynamic model revision techniques based on surrogate model, Automotive Engineering, 36(4), pp. 448-452. DOI: 10.19562/j.chinasae.qcgc.2014.04.012.

[14] Simpson, T. W., Maucry, T. M., Korte, J. J., et al. (2001). Kriging models for global approximation in simulation-based multidisciplinary design optimization, AIAA J, 39(12), pp. 2233-2241. DOI: 10.2514/3.15017.

[15] Simpson, T., Mistree, F., Korte, J., et al. (1998). Comparison of response surface and kriging models for multidisciplinary design optimization, in Aiaa Paper 7 ThAiaa/usaf/nasa/issmo Symposium on Multidisciplinary Analysis \& Optimization, 98, 4755. DOI: 10.2514/6.1998-4755.

[16] Wang, J., Wang, C., Zhao, J. (2017). Frequency response function-based model updating using Kriging model, Mechanical Systems \& Signal Processing, 87, pp. 218-228. DOI: 10.1016/j.ymssp.2016.10.023.

[17] Martin, J. D., Simpson, T. W. (2004). On the use of Kriging models to approximate deterministic computer models, in ASME 2004 International Design Engineering Technical Conferences and Computers and Information in Engineering Conference. American Society of Mechanical Engineers. Utah, USA. September 28- October 2.

[18] Gano, S., Renaud, J., Martin, J., et al. (2005). Update strategies for Kriging models for use invariable fidelity optimization, Struct. Multidiscip. Optim, 32(4), pp. 287-298. DOI: 10.1007/s00158-006-0025-y.

[19] Jin, R., Chen, W., Sudjianto, A. (2004). An efficient algorithm for constructing optimal design of computer experiments, Journal of Statistical Planning \& Inference, 134(1), pp. 268-287. DOI: 10.1016/j.jspi.2004.02.014.

[20] Fang, K. T., Ma, C. X., Winker, P. (1999). Centered L2-discrepancy of random sampling and Latin hypercube design, and construction of uniform designs, Mathematics of Computation, 71 (237), pp. 275-296.

DOI: 10.1090/S0025-5718-00-01281-3.

[21] Ahmadian, H., Mottershead, J. E., Friswell, M. I. (1998). Regularisation methods for finite element model updating, Mechanical Systems \& Signal Processing, 12(1), pp. 47-64. DOI: 10.1006/mssp.1996.0133. 